\title{
Die Wirtschafts- und Finanzkrise 2008/09 - die erste Bewährungsprobe für die Europäische Wirtschafts- und Währungsunion
}

\author{
André Schmidt*
}

Im April 2009 stellte der Internationale Währungsfonds (IWF) fest, dass die von den USA ausgehende Finanzkrise, die Weltwirtschaft in einem bisher noch nicht vorstellbaren Ausmaß treffen wird. ${ }^{1}$ Insbesondere in den etablierten Industriestaaten sei mit erheblichen rezessiven Tendenzen zu rechnen. Davon sind, neben den USA und Japan, vor allem auch die europäischen Volkswirtschaften betroffen. Die mit der Rezession einhergehende Gefahr des Entstehens von Massenarbeitslosigkeit durch massive Überkapazitäten und Produktionsrückgänge ist geeignet, die ökonomische Entwicklung Europas nachhaltig zu gefährden. Daher erweist sich die Wirtschafts- und Finanzkrise als erste ernsthafte Bewährungsprobe für die Wirtschafts- und Währungsunion (WWU).

Für Ökonomen und Politikwissenschaftler ist dies ein Feldversuch erster Güte, kann doch die WWU zum ersten Mal beweisen, ob die Argumente, die für ihre Legitimation vorgetragen wurden - Europa wirtschaftlich unabhängiger von der Entwicklung der Weltmärkte werden zu lassen und damit die Möglichkeiten einer eigenen Stabilisierungspolitik zu stärken -, einer Überprüfung standhalten können. Mit Eintreten der Krise kann die WWU zeigen, inwieweit die bisherigen Integrationsschritte tatsächlich ausreichen, eine weltweite Rezession, die in ihrer Entwicklung durchaus Parallelen zur Großen Depression enthält, ${ }^{2} \mathrm{zu}$ absorbieren und durch eine gemeinschaftliche Geldpolitik und wirtschaftspolitische Koordination die drohende Rezession zu lindern. Ziel dieses Aufsatzes ist es, die Reaktionen der Europäischen Union und ihrer Institutionen retrospektiv auf die Wirtschafts- und Finanzkrise darzustellen und damit der Frage nachzugehen, ob die WWU ihre ,Feuertaufe ‘ bestanden hat. Es ist sicherlich noch zu früh, ein abschließendes Urteil zu bilden, daher kann dies allenfalls als ein erster Versuch einer Analyse der Krisenreaktionsfähigkeit der WWU angesehen werden.

Bezüglich der wirtschaftspolitischen Reaktionen auf die Krise kann zwischen geld- und fiskalpolitischen Handlungsfeldern unterschieden werden. Da die Ausgestaltung der Geldpolitik der unabhängigen Europäischen Zentralbank (EZB) unterliegt, wird zunächst auf die geldpolitischen Reaktionen der Zentralbank eingegangen. Die Geldpolitik steht auch deswegen an erster Stelle, weil der Währungspolitik im Rahmen der wirtschaftlichen Integration schon immer eine besondere Rolle zukam. Bekanntlich betraf die Subprime-Krise zunächst den Banken- und Finanzsektor, wodurch vor allem geldpolitisches Eingreifen erforderlich war. Erst mit der Gefahr zunehmender Spill-over-Effekte vom monetären in den realen Sektor wurde mehr und mehr fiskalpolitischer Einsatz der Regierungen der Mitgliedstaaten sowie deren Koordination durch die Europäische Union erforderlich. Diese fiskalpolitischen Aktivitäten und deren Koordination sind im dritten Teil Gegenstand der Analyse. Eine ab-

\footnotetext{
* Prof. Dr. André Schmidt, Lehrstuhl für Makroökonomik und Internationale Wirtschaft, Universität Witten/ Herdecke.

1 Internationaler Währungsfonds: World Economic Outlook. Crises and Recovery, April 2009.

2 Albrecht Ritschl: War 2008 das neue 1931?, in: Aus Politik und Zeitgeschichte 20/2009, S. 27-32, hier S. 31.
} 
schließende Bewertung einschließlich eines perspektivischen Ausblicks sollen dann mögliche Stärken und Schwächen im bisherigen Gefüge der WWU offenlegen.

\section{Monetäre Reaktionen und die Rolle der EZB}

\section{Die Wirtschafts- und Finanzkrise als Herausforderung an die Geldpolitik}

Der Geldpolitik kam seit Krisenausbruch eine Schlüsselrolle zu. Da im Zuge der Großen Depression vor allem die zu restriktive Geldpolitik der Notenbanken als Hauptursache für Ausbreitung und schwerwiegende Folgen der Krise erkannt worden war, ${ }^{3}$ sollte nun vor allem durch eine stark expansive Geldpolitik von vornherein eine Verschärfung der Krise verhindert werden. Das heißt: Spätestens seit dem akuten Eintreten der Wirtschafts- und Finanzkrise rückte die Geldpolitik wieder in das Zentrum der wirtschaftspolitischen Diskussionen. Es galt, den drohenden Kollaps auf den Finanzmärkten mittels einer expansiven und primär liquiditätszuführenden Geldpolitik zu verhindern. Der aggressiven Strategie der US-Fed folgte auch die EZB mit einer zwar moderateren aber ebenfalls expansiv ausgerichteten Geldpolitik. Seither hat jedoch die Diskussion unter den Ökonomen darüber, inwieweit diese Geldpolitik tatsächlich geeignet ist, zu einer wesentlichen Stabilisierung beizutragen, neue Nahrung erhalten. Insbesondere in den USA wird kontrovers darüber diskutiert, inwieweit die Strategie der Fed tatsächlich zur Stabilisierung beitragen kann oder ob nicht hier das Fundament für eine weitere Krise gelegt wird. Die Argumentationslinien auf beiden Seiten lassen sich wie folgt zusammenfassen:

Die Seite der Kritiker wird angeführt von John B. Taylor. ${ }^{4}$ Er sieht insbesondere in der stark expansiven Geldpolitik der Fed in den vergangenen Jahren den Hauptauslöser für das Zusammenbrechen des US-amerikanischen Häusermarktes und den daraus entstandenen Folgewirkungen. „No Boom, no bust“, so lautet das Ergebnis seiner Analyse. Als Ursachen für die sich daran anschließende Bankenkrise kommen zwei Möglichkeiten in Betracht. Zum einen kann es sich um eine akute Liquiditätskrise handeln, die durch , bank runs ‘ ausgelöst wird, zum anderen kann ein dramatisch ansteigendes Ausfallrisiko auf dem Kreditmarkt, begleitet von einem kollabierenden Interbankenmarkt, als mögliche Krisenursache ausgemacht werden. Während die Liquiditätskrise vor allem in der Großen Depression als der Hauptauslöser angesehen werden kann, ist die aktuelle Krise hauptsächlich durch das drastisch gestiegene Ausfallrisiko, einhergehend mit einem gravierenden Vertrauensschwund, insbesondere im Interbankenhandel, gekennzeichnet. Taylor sieht insbesondere in dem starken Anstieg des Zinsspreads zwischen LIBOR und dem Reposatz ${ }^{5}$ der Fed einen wesentlichen Beleg für die Dominanz des Ausfallrisikos. Wenn nun jedoch tatsächlich das Ausfallrisiko der dominierende Auslöser der Finanzkrise ist, dann vermag - so Taylor - eine aggressive Geldpolitik gar nichts zu bewirken. Vielmehr müssten Maßnahmen ergriffen werden, die das Vertrauen insbesondere zwischen den Banken wieder stärken.

3 Milton Friedman/Anna Jacobson Schwartz: The Great Contraction 1929-1933, Princeton 1967, S. 25.

4 John B. Taylor: The Financial Crisis and the Policy Responses: An Empirical Analysis of What Went Wrong, National Bureau of Economic Research: NBER Working Paper Nr. 14631, Januar 2009. Vgl. hierzu auch Olivier Blanchard: The Crisis: Basic Mechanisms, and Appropriate Policies, Internationaler Währungsfonds: IMF Working Paper 80/2009.

5 LIBOR steht für ,London Interbank Offered Rate ' und ist ein Referenzzinssatz, der täglich einen Mittelwert der Zinssätze angibt, zu denen Banken am Londoner Geldmarkt bereit sind, kurzfristige unbesicherte Finanzgeschäfte im Interbankenmarkt zu tätigen. Der Reposatz ist ein Zinssatz für (besicherte) Pensionsgeschäfte (Repurchase Agreement, kurz Repo). 
Auf der anderen Seite argumentiert vor allem Frederic S. Mishkin, ${ }^{6}$ dass die derzeitig expansive Geldpolitik der Fed und auch der EZB alternativlos sei. Er verweist insbesondere darauf, dass trotz der erheblichen Zinssenkungen die Finanzierungskosten zum Ende des vergangenen Jahres aufgrund der Risikoaufschläge sehr stark gestiegen sind. Mishkin teilt die Argumentation von Taylor insoweit, dass auch für ihn die gestiegenen Zinsspreads Ausdruck für das höhere Ausfallrisiko sind und dieses durch eine expansive Geldpolitik kaum reduziert werden kann. Jedoch solle man nicht nur das Ausfallrisiko berücksichtigen; vielmehr komme es vor allem auf das makroökonomische Risiko an. Damit ist gemeint, dass in Phasen rezessiver Wirtschaftsentwicklung die am Markt bestehende Unsicherheit zwischen den Akteuren wächst. Je stärker diese Unsicherheit zunimmt, desto größer wird wiederum das Bewertungsrisiko, wodurch sich auch indirekte Wirkungen für das Ausfallrisiko ergeben. Da die aktuelle geldpolitische Strategie der Zentralbanken dieses makroökonomische Risiko effektiv senkt, sei diese Art der Geldpolitik ohne Alternative. Wichtig sei nur, dass die Glaubwürdigkeit der Zentralbanken nicht beschädigt wird, um die zukünftigen Inflationserwartungen möglichst gering zu halten.

Vor dem Hintergrund dieser ambivalenten Beurteilung adäquater geldpolitischer Antworten ist auch die Strategie der EZB zu bewerten. Auf der einen Seite galt es, zunächst mithilfe einer expansiven Geldpolitik den Märkten ein entschlossenes Handeln zu demonstrieren und das Zusammenbrechen der Finanzmärkte zu verhindern. Auf der anderen Seite war jedoch auch Zurückhaltung und Besonnenheit angebracht, um nicht zu früh sämtliche Instrumente einzusetzen und sich dem Risiko eines späteren Verlustes der Handlungsfähigkeit auszusetzen sowie nicht unnötig schon jetzt zukünftige Inflationserwartungen zu schüren. Daher war von vornherein eine besonnene geldpolitische Strategie notwendig. Dies mag auch eine Erklärung für die deutlich zurückhaltende Politik der EZB im Vergleich zur Fed sein.

\section{Die Geldpolitik der EZB von Oktober 2008 bis August 2009}

Bereits mit dem Auftreten der ersten Spannungen auf dem Euro-Geldmarkt im August 2007 reagierte die EZB mit einer zusätzlichen, befristeten Bereitstellung von Liquidität. Zunächst wurden die Banken zu Beginn der Mindestreserve-Erfüllungsperiode großzügig mit Liquidität versorgt, um dann die Liquiditätszufuhr nach unten anzupassen, sodass sich der Liquiditätsüberschuss der Banken zum Ende der Erfüllungsperiode wieder im Durchschnitt auf nahezu Null belief. Zur weiteren Stabilisierung des Euro-Geldmarktes begann die EZB mit der Durchführung längerfristiger Refinanzierungsgeschäfte, zunächst mit einer Laufzeit von drei und später von sechs Monaten.

Die Insolvenz der US-amerikanischen Investmentbank „Lehman Brothers“ führte dann im September 2008 zu einer nochmals erheblichen Verschlechterung der Lage auf den EuroGeldmärkten. Daher kündigte die EZB unmittelbar im September 2008 die Durchführung eines Refinanzierungsgeschäfts mit einer Sonderlaufzeit von 38 Tagen an, wodurch 120 Milliarden Euro zugeteilt werden konnten. Allerdings reichten diese Maßnahmen noch nicht aus, um die Lage auf den Euro-Geldmärkten zu entspannen. Nach dem Zusammenbruch der US-amerikanischen Investmentbank „Lehman Brothers“ bestand die reale Furcht vor weiteren schweren Bankzusammenbrüchen, die auch vor dem europäischen Markt nicht haltmachen würden. Im Hintergrund standen Bedenken hinsichtlich der finanziellen Solidität einiger anderer Finanzinstitutionen sowohl in den Vereinigten Staaten als auch in Europa.

6 Frederic S. Mishkin: Is Monetary Policy Effective during Financial Crises?, in: American Economic Review 2/2009, S. 573-577. 
Aufgrund dessen kam der Euro-Geldmarkt nahezu vollkommen zum Erliegen. Mit einem ersten Schritt senkte die EZB zunächst den Mindestbietungssatz für Hauptrefinanzierungsgeschäfte.

Von größerer Bedeutung als die allgemeine Zinssenkungspolitik, die in Abstimmung mit allen anderen wichtigen Zentralbanken der Welt erfolgte, war jedoch die Änderung des Tenderverfahrens.

Das ,Tenderverfahren` bezeichnet die technische Abwicklung der Offenmarktpolitik der EZB. Im Wesentlichen handelt es sich hierbei um die Festlegung der Konditionen, zu denen sich die Geschäftsbanken in einem Auktionsverfahren - dem sogenannten Tender - bei der Zentralbank refinanzieren können. Dabei lassen sich vereinfacht zwei Formen unterscheiden: das Mengentenderverfahren, bei dem die Zentralbank den Zinssatz vorgibt und eine Mengenzuteilung vornimmt, und das sogenannte Zinstenderverfahren, bei dem die Geschäftsbanken in ihren Geboten sowohl Mengen- als auch Zinsgebote abgeben müssen.

Angesichts des Ausbruchs der Krise wechselte die EZB nun vom Zinstenderverfahren zum Mengentenderverfahren mit der Besonderheit, dass zum vorgegebenen Zinssatz eine vollständige Zuteilung erfolgt. Das heißt: Jede Geschäftsbank kann sich nun zu dem von der EZB geforderten Zinssatz zur jeweils gewünschten Menge bei der EZB refinanzieren, sofern sie über genügend notenbankfähige Sicherheiten verfügt. ${ }^{7}$

Mit diesem wichtigen Schritt konnte die EZB zwei Vorteile realisieren. Zum einen konnte sie sicherstellen, dass alle Banken bei entsprechendem Bedarf ausreichend mit Liquidität versorgt werden konnten, und zum anderen konnte damit das Eurosystem bestehende Zweifel über die Liquiditätszuteilung einzelner Banken ausräumen, da eine vollständige und garantierte Zuteilung erfolgte.

Das Maßnahmenpaket vom 8. Oktober 2008 enthielt noch eine dritte wichtige Komponente. Die EZB senkte die Zinssätze der ständigen Fazilitäten (Spitzenrefinanzierungsfazilität und Einlagenfazilität), zu denen die Geschäftsbanken über Nacht Refinanzierungsmittel erwerben oder überschüssige Liquidität anlegen können und reduzierte den Korridor zwischen diesen beiden Zinssätzen von bisher 200 auf 100 Basispunkte. Dadurch gewann die Einlagenfazilität für Geschäftsbanken mit Liquiditätsüberschüssen im Vergleich zum Interbankenmarkt erheblich an Attraktivität, wodurch das Eurosystem den zu dieser Zeit stark beschädigten Geldmarkthandel ersetzte.

Mitte Oktober wurden diese Maßnahmen noch durch eine Ausweitung der notenbankfähigen Sicherheiten und durch weitere liquiditätszuführende Feinsteuerungsmaßnahmen ergänzt. Bezüglich der Ausweitung notenbankfähiger Sicherheiten wurde deren Bonitätsschwellenwert von ,A-' auf ,BBB-“8 ${ }^{\circ}$ gesenkt (ausgenommen blieben davon , asset-backed securities', also Wertpapiere, die mit bisher nicht liquidierbaren Forderungen besichert sind). Die liquiditätszuführenden Feinsteuerungsoptionen betrafen insbesondere Fremdwährungsgeschäfte in US-Dollar und Schweizer Franken. Mithilfe dieser Feinsteuerungsoptionen sollten zum einen Liquiditätsengpässe bei der Versorgung mit US-Dollar und Schweizer Franken vermieden werden, zum anderen sollte damit auch eine wechselkursstabilisierende Wirkung erzielt werden.

Aufgrund der Umstellung des Tenderverfahrens mit vollständiger Zuteilung konnten die Geschäftsbanken ihren gesamten Liquiditätsbedarf über die wöchentlichen Tender der EZB

7 Europäische Zentralbank: Die Umsetzung der Geldpolitik seit August 2007, in: Europäische Zentralbank (Hrsg.): Monatsbericht Juli 2009, Frankfurt am Main 2009, S. 85-100.

8 Ratings sind Einstufungen der Bonität von Kreditnehmern durch private Ratingagenturen. Ein ,AAA'-Rating spiegelt dabei die Einschätzung wider, dass das bewertete Unternehmen über eine sehr hohe Bonität verfügt, während ein ,BBB-'-Rating im unteren Bereich einer als durchschnittlich eingeschätzten Bonität liegt. 
decken. Dadurch wurden die Unsicherheit im Markt und insbesondere das gestiegene Ausfallrisiko im Interbankenhandel erheblich reduziert. Die Liquiditätsversorgung wurde nun nicht mehr von der EZB bestimmt, sondern ausschließlich durch die Geldnachfrage der Geschäftspartner. Dies hat einen Zusammenbruch des Interbankenmarktes wirkungsvoll verhindert. Die Umstellung auf den Mengentender mit vollständiger Zuteilung sowie die Verringerung des Korridors der ständigen Fazilitäten waren beispiellose Schritte in der noch jungen Geschichte der Geldpolitik der EZB. Durch das schnelle und entschlossene Handeln der EZB konnte somit größerer Schaden abgewendet werden. Für den Erfolg dieser Maßnahmen spricht auch, dass die EZB bereits im Januar 2009 den Fazilitätenkorridor wieder auf die alte Relation von 200 Basispunkten ausweiten konnte.

Weitere Zinssenkungen und die Beibehaltung des Mengentenders mit vollständiger $\mathrm{Zu}-$ teilung bis zur Gegenwart haben nachdrücklich zu einer Stabilisierung der Situation beigetragen. Dies hat auch dazu geführt, dass sich die Finanzierungskosten der Wirtschaft seither reduziert haben. So sind die kurzfristigen nominalen Kreditzinsen der Banken für Großkredite an nicht zum Finanzsektor gehörende Kapitalgesellschaften zwischen Oktober 2008 und April 2009 um rund 300 Basispunkte gesunken. ${ }^{9}$ Die Tatsache, dass sich für einzelne Unternehmen die Finanzierungskosten dennoch erhöht haben, liegt weniger an der geldpolitischen Reaktion der EZB als vielmehr an der gesunkenen Bonität der Unternehmen aufgrund der sich zunehmend verschlechternden realwirtschaftlichen Bedingungen.

Vergleicht man die geldpolitischen Reaktionen der EZB mit denen der Fed, so lässt sich feststellen, dass die Fed ihre Strategie zugunsten des , quantitative easings ‘ änderte. ,Quantitative easing ' bedeutet, dass die Zentralbank in der Situation eines Leitzinssatzes von nahezu Null Prozent versucht, die Geldpolitik noch weiter zu lockern. Ab März 2009 kündigte die Fed an, Staatsanleihen (,treasury bills') sowie immobilienmarktnahe Wertpapiere (, government-sponsored enterprise debts' ${ }^{\prime 0}$ und ,mortgage-backed securities ${ }^{\text {'11) }}$ im Umfang von über einer Billion US-Dollar aufzukaufen, was faktisch der Strategie des ,quantitative easings ' entspricht. ${ }^{12}$ Die sich daraus unmittelbar ergebenden Geldmengeneffekte führen zu einem deutlich höheren Inflationspotenzial, da diese Liquidität im Gegensatz zu Refinanzierungsgeschäften nur langfristig wieder aus dem Markt herausgenommen werden kann. Demgegenüber lässt sich die geldpolitische Reaktion der EZB eher als ein ,credit easing ' bezeichnen, das vor allem auf die Gestaltung günstigerer Refinanzierungsbedingungen der Geschäftsbanken abstellt.

Zusammenfassend lässt sich konstatieren, dass die EZB ihre ,Feuertaufe ' bestanden hat. Aufgrund der oben beschriebenen Maßnahmen wurde den Finanzmarktteilnehmern signalisiert, dass hinsichtlich der Verfügbarkeit von Zentralbankliquidität kein Anlass zur Sorge bestand. Damit wurde ein wichtiges vor allem vertrauensbildendes Signal an die Marktteilnehmer gesendet. Gleichzeitig hat die EZB jedoch auch ausreichend Vorsicht walten lassen.

9 Europäische Zentralbank: Ergebnisse der Umfrage zum Kreditgeschäft im Euro-Währungsgebiet vom April 2009, in: Europäische Zentralbank (Hrsg.): Monatsbericht Mai 2009, Frankfurt am Main 2009, S. 23-31, hier S. 23.

10 ,Government-sponsored enterprises ‘ sind Unternehmen in Privatbesitz, die durch den US-Kongress errichtet werden, um die Effizienz der Kapitalmärkte zu verbessern. Sie verfügen über bestimmte Privilegien und sind in ihrer Arbeit dafür aber bestimmten vom US-Kongress festgelegten Beschränkungen und Zielen unterworfen beziehungsweise verpflichtet. Vgl. Kevin R. Kosar: Government-Sponsored Enterprises (GSEs): An Institutional Overview, CRS Report for Congress, Order Code RS21663, Washington D.C. 2007.

11 ,Mortgage-backed securities ' sind ein Typus von , asset-backed securities', die mit Hypothekenkrediten besichert sind.

12 Richard G. Anderson: The Curious Case of the U.S. Monetary Base, in: The Regional Economist 3/2009, S. 12-13. 
Sie hat ihre Maßnahmen sorgfältig ausgewählt und darauf geachtet, sich weitere Handlungsoptionen, wie Zinssenkungen oder Feinsteuerungsoptionen zu erhalten, wodurch niemals der Eindruck entstand, dass sämtliche Maßnahmen zur Stabilisierung ausgeschöpft wurden. Auch dies hat wesentlich zu weiteren Reputationsgewinnen der Zentralbank beigetragen.

\section{Fiskalpolitische Reaktionen und die Rolle der Mitgliedstaaten}

Weniger einheitlich stellten sich dagegen die Strategien der Mitgliedstaaten bei der Bekämpfung der sich aus der Finanzkrise resultierenden Wirtschaftskrise dar. Dabei standen die Mitgliedstaaten im Herbst 2008 vor einer doppelten Herausforderung. Zum einen mussten sie dafür Sorge tragen, dass jeweils ihr inländischer Bankenmarkt neben dem aktiven Eingreifen der Zentralbank auch durch inländische wirtschaftspolitische Schritte stabilisiert wird, und zum anderen mussten sie bereits sehr früh versuchen, ein Abgleiten der konjunkturellen Situation in eine Rezession zu verhindern. Dabei darf nicht verschwiegen werden, dass die Ökonomien der Mitgliedstaaten der Europäischen Union sehr unterschiedlich von der Krise betroffen wurden. Während Spanien und Großbritannien sich zunächst einer starken Krise auf dem Immobilienmarkt ausgesetzt sahen, brach insbesondere in Deutschland und Frankreich die Exportnachfrage stark ein. Da die Länder in unterschiedlichen Intensitäten und in unterschiedlichen Bereichen von der Krise betroffen waren, war es kein Wunder, dass ein einheitliches Vorgehen zunächst nicht oder nur schwer realisierbar war. Auffallend jedoch war in einigen Ländern der protektionistische Unterton mit dem im ersten Reflex auf die Krise reagiert wurde.

Am 26. November 2008 legte die Europäische Kommission einen ersten Entwurf für ein gesamteuropäisches Konjunkturprogramm vor, ${ }^{13}$ welches die Grundlage für die Europäische Ratstagung vom Dezember 2008 darstellte. ${ }^{14}$ Im Mittelpunkt standen zunächst der Schutz und die Erhaltung von Arbeitsplätzen sowie die Umsetzung zukunftsträchtiger Investitionen im Rahmen der Lissabon-Strategie. Der fiskalische Impuls belief sich auf circa 200 Milliarden Euro, von denen 170 Milliarden Euro aus den nationalen Haushalten und 30 Milliarden Euro von der Europäischen Investitionsbank stammten. Ein Konflikt mit dem Stabilitätsund Wachstumspakt wurde nicht gesehen, da aufgrund der Reform im Jahr 2005 der Pakt ausreichende Flexibilität ermöglicht: Ein Abweichen vom Drei-Prozent-Defizitkriterium gilt nach der Reform bereits dann als zulässig, wenn die wirtschaftliche Wachstumsrate deutlich unter der Trendwachstumsrate liegt. ${ }^{15}$ Diese Bedingung dürfte aufgrund der Wirtschaftsund Finanzkrise für alle Volkswirtschaften innerhalb der Europäischen Union erfüllt sein.

Die Umsetzung der konjunkturstützenden Maßnahmen erfolgte auf der Basis der Lockerung der Vergabekriterien für den Europäischen Sozialfonds und durch vorgezogene Auszahlungen im Rahmen des Kohäsionsfonds. Die Verwendung der Mittel des Konjunkturprogramms lag in der Kompetenz der Mitgliedstaaten.

Eine wesentliche Rolle bei der Sicherung der von der Wirtschaftskrise betroffenen Arbeitnehmer kam dabei dem sogenannten Globalisierungsfonds zu. Hierbei wurde die Möglichkeit der Nutzung des Globalisierungsfonds bereits für Entlassungen ab 500 Arbeitnehmern geöffnet,

13 Europäische Kommission: Umfassendes Konjunkturprogramm der Kommission für Wachstum und Beschäftigung zur Ankurbelung der Nachfrage und Wiederherstellung des Vertrauens in die europäische Wirtschaft, Pressemitteilung, IP/08/1771, 26.11.2008; Europäische Kommission: Mitteilung der Kommission an den Europäischen Rat. Europäisches Konjunkturprogramm, KOM (2008) 800.

14 Rat der Europäischen Union: Tagung des Europäischen Rates vom 11./12. Dezember 2008 in Brüssel. Schlussfolgerungen des Vorsitzes, Dok. 17271/1/08.

15 Karl Heinz Hausner: Der neue Stabilitäts- und Wachstumspakt und die deutsche Staatsverschuldung, in: Wirtschaftsdienst 4/2005, S. 238-243, hier S. 240. 
die Förderdauer von 12 auf 24 Monate erweitert und der EU-Finanzbeitrag von 50 auf 75 Prozent erhöht. ${ }^{16}$ Im Rahmen dieses Maßnahmenpaketes handelte es sich vor allem um Sofortmaßnahmen, die noch keiner größeren und aufwendigeren Koordinierung bedurften. Im Mittelpunkt stand die Einbettung europäischer Förderinstitute in die nationalen Konjunkturprogramme.

Dennoch ist es für die Koordination der Konjunkturprogramme wichtig, dass die nationalen Konjunkturprogramme nicht nur von den großen Volkswirtschaften der Europäischen Union finanziert und aufgelegt werden, sondern auch von den kleineren Mitgliedstaaten. Für die kleineren Staaten ergibt sich grundsätzlich ein Anreiz zum Free-rider-Verhalten. Sie selbst profitieren von den positiven Spill-over-Effekten in Form einer höheren Exportnachfrage aus den größeren Volkswirtschaften und haben daher nur einen geringen Anreiz, sich an den Kosten der antizyklischen Konjunkturprogramme zu beteiligen. Mithilfe des oben beschriebenen Trittbrettfahrerverhaltens lässt sich auch die empirische Evidenz begründen, warum eher die größeren Volkswirtschaften gegen den Stabilitäts- und Wachstumspakt verstoßen als die kleineren Volkswirtschaften. ${ }^{17}$ Die kleineren Länder profitieren von der verschuldungsfinanzierten expansiven Fiskalpolitik größerer Volkswirtschaften durch eine höhere Exportnachfrage und haben daher nur geringe Anreize, selbst eine verschuldungsfinanzierte expansive Fiskalpolitik zu betreiben. Diese Beobachtung lässt sich auch auf die Gegebenheiten der Wirtschafts- und Finanzkrise übertragen. Mit der Vereinbarung, dass alle Mitgliedstaaten 1,2 Prozent ihres nationalen Bruttoinlandsprodukts (BIP) als konjunkturellen Impuls verwenden, wurde dieses Koordinationsproblem geradezu mustergültig gelöst.

Zu Beginn des Jahres 2009 wurde dennoch zunehmend deutlich, dass eine bessere gesamteuropäische Koordinierung der nationalen Anstrengungen erforderlich war. So erkannten die Mitgliedstaaten, dass ihre ersten Sofortmaßnahmen noch nicht ausreichten und legten daher neue, verfeinerte Konjunkturprogramme auf, weiterhin erkannten sie die Notwendigkeit der Ausnutzung der Vorteile einer gebündelten Vorgehensweise. Auf der Basis dieser Erfahrungen wurden auf der Ratssitzung im März 2009 in Brüssel weitere Maßnahmen und Beschlüsse zur Stabilisierung der Wirtschaft verabschiedet. Diese umfassten einerseits Vorschläge zur Stabilisierung der Finanzmärkte und deren effizienter Regulierung sowie die Durchführung der Konjunkturprogramme. Hierbei wurde auf die Fortführung und Intensivierung der Beschlüsse vom Dezember 2008 verwiesen. Zusätzliche konjunkturelle Impulse sollen durch zielgerichtete Investitionen in die Versorgungssicherheit des Energiesektors und durch die Anbindung ländlicher Räume an das Breitbandinternet in Höhe von 5 Milliarden Euro erreicht werden. Die Europäische Investitionsbank stockt ihre Darlehensfazilitäten für kleine und mittlere Unternehmen um 15 Milliarden Euro auf. Ausdrücklich wurden die Mitgliedstaaten noch einmal darauf hingewiesen, dass der Verwirklichung des Binnenmarktes bei der Krisenbewältigung oberste Priorität zukommt. Mit anderen Worten sollten die Mitgliedstaaten bei dem Einsatz ihrer nationalen Konjunkturprogramme vermehrt auf die gesamteuropäische Dimension des Binnenmarktes Rücksicht nehmen. ${ }^{18}$ Dies zeigt, dass die Mitgliedstaaten in der Krise durchaus geneigt waren, nationalen Interessen gegenüber gesamteuropäischen Interessen den Vorrang einzuräumen. Gleichzeitig wurde die Notwendigkeit der Fortführung des LissabonProzesses über das Jahr 2010 hinaus zur Sicherung der nachhaltigen Wachstumsperspektiven für die Volkswirtschaften der Europäischen Union bekräftigt.

16 Europäische Kommission: Globalisierungsfonds: Unterstützung für mehr entlassene Arbeitnehmerinnen und Arbeitnehmer, Pressemitteilung, IP/08/1985, 16.12.2008.

17 Bodo Herzog: Warum verstoßen vorwiegend die großen EWU-Länder gegen den Stabilitäts- und Wachstumspakt?, in: Vierteljahreshefte zur Wirtschaftsforschung 3/2004, S. 405-417, hier S. 410.

18 Europäische Kommission: Mitteilung für die Frühjahrstagung des Europäischen Rates. Impulse für den Aufschwung in Europa. Teil 1, KOM (2009) 114. 


\section{Bewertung und Ausblick}

Bei der Beurteilung der Reaktionen der Europäischen Union auf die Wirtschafts- und Finanzkrise ist zwischen monetären und fiskalischen Reaktionen zu unterscheiden. Was die Reaktionen im monetären Bereich betrifft, so kann man der EZB ein sehr maßvolles und der Situation angemessenes Verhalten attestieren. Die liquiditätszuführenden Maßnahmen konnten insbesondere den Geldmarkt wirkungsvoll stabilisieren. Gleichzeitig hat die Zentralbank durch ihre Zinsschritte nicht nur die Refinanzierungsmöglichkeiten der Geschäftsbanken verbessert, sondern auch die Finanzierungsmöglichkeiten einzelner Unternehmen. Damit konnten zumindest die Folgen der Krise abgemildert werden. Positiv ist auch die Stringenz des geldpolitischen Instrumenteneinsatzes hervorzuheben. Die EZB hat stets auf den Märkten den Eindruck hinterlassen, dass sie jederzeit handlungsfähig ist und ihre Reaktionsfähigkeit zu keiner Zeit infrage gestellt war. Dies hat das Eurosystem ohne Frage attraktiver auch für solche Staaten werden lassen, die bisher noch nicht Mitglied der Europäischen Union sind (beispielsweise Island) oder noch nicht den Euro eingeführt haben. Insgesamt hat die EZB durch ihre Maßnahmen ihre Reputation weiter stärken und das Vertrauen in das Gesamtsystem ausbauen können. Gerade in der gegenwärtigen Krise hat sich die Währungsunion als ein wichtiger Stabilitätsanker erwiesen. Hätte es die Währungsunion nicht gegeben, wäre der Abwertungsdruck auf einzelne Währungen in Europa sehr viel größer geworden, was zu noch viel stärkeren Ungleichgewichten und Marktverwerfungen hätte führen können. Daher lässt sich, was die Währungsunion betrifft, konstatieren, dass diese im Augenblick der Krise vor allem aufgrund des verantwortungsvollen Umgangs der EZB die an sie zu stellenden Anforderungen erfüllt hat.

Die nächste Herausforderung im monetären Bereich wird es nun sein, inwieweit es der EZB gelingt, entschieden möglichen Inflationserwartungen entgegenzutreten. Allerdings dürften hierbei die bisherigen Reputationsgewinne von Vorteil sein. Sollte die EZB - unter der Voraussetzung, dass sich bis Dezember 2009 die Lage an den Finanzmärkten weiter entspannt - den Mengentender mit garantierter Liquiditätszuteilung einstellen, sollte sie auch wieder schnell in der Lage sein, überschüssige Liquidität am Markt abzuschöpfen, da mit Auslaufen der Tendergeschäfte die Geldmenge verringert werden kann. Da die EZB auf ein ,quantitative easing 'verzichtet hat, kann die drohende Gefahr zukünftig hoher Inflationsraten aufgrund einer zu hohen Geldmenge relativiert werden.

Was die Beurteilung der fiskalpolitischen Maßnahmen betrifft, so ist es zum gegenwärtigen Zeitpunkt außerordentlich schwierig, ihren Erfolg zu evaluieren. Grundsätzlich fällt jedoch auf, dass es hier auf Gemeinschaftsebene nur geringe Ansätze zu einer konsistenten Strategie gab. Dies muss jedoch zunächst kein Nachteil sein, da es außerordentlich fraglich ist, ob die Gemeinschaft bei einer harmonisierten Vorgehensweise bessere Erfolge erzielen kann als die einzelnen Mitgliedstaaten unter Verantwortung ihrer nationalen Parlamente. Dies gilt umso mehr, als die Wirtschafts- und Finanzkrise die Mitgliedstaaten sehr unterschiedlich getroffen hat, was ein wichtiges Argument für eine dezentrale fiskalpolitische Steuerung liefert.

Ein zentralisiertes Vorgehen ist auch aus der Sicht des Subsidiaritätsprinzips nicht zwingend erforderlich. Nur im Fall gemeinschaftsweiter positiver Externalitäten ist ein zentralisiertes Konjunkturprogramm erforderlich. Aufgrund der unterschiedlichen Wirtschaftstrukturen und der Unterschiedlichkeit der betroffenen Sektoren ist die Umsetzung der konjunkturellen Hilfe primär auf der Ebene der Mitgliedstaaten zu sehen. Richtig war es jedoch, dafür Sorge zu tragen, dass sich alle Mitgliedstaaten an den Konjunkturprogrammen in angemessener Höhe (1,2 Prozent des nationalen BIP) beteiligen um das beschriebene 
Trittbrettfahrerverhalten auszuschließen. Was die inhaltliche Festlegung der von der Gemeinschaft formulierten konjunkturellen Hilfen betrifft, so blieben diese doch eher vage und haben überwiegend deklaratorischen Charakter. Dies betrifft die Aspekte des Binnenmarktprogramms sowie den Verweis auf übliche Schlüsseltechnologien wie Energie und Breitbandinternet. Inwieweit diese tatsächlich geeignet sind, darf vor dem Hintergrund der bisherigen industriepolitischen Erfahrungen in der Europäischen Union bezweifelt werden. Insgesamt betrachtet scheint auch der Verweis auf die Wirkungen der Lissabon-Strategie wenig Erfolg versprechend. Das ehrgeizige Vorhaben von einst, die Europäische Union zum dynamischsten wissensbasierten Wirtschaftsraum der Welt zu generieren, ${ }^{19}$ musste bereits in den vergangenen Jahren mehrfach relativiert werden. Daher ist kritisch zu fragen, unter welchen Umständen nun eine neue Offensive zu mehr Erfolg als in der Vergangenheit führen soll.

Grundsätzlich kann jedoch nicht bestritten werden, dass die Europäische Union eine neue, langfristige koordinierte Wachstumsstrategie benötigt. Während die Maßnahmen der Fiskalpolitik nur kurzfristig wirken, müssen die langfristigen Wachstumskräfte gestärkt werden. Dazu bedarf es einer Strategie, die sich weniger an quantitativen Vorgaben zu Beschäftigungsquoten und Produktivitätskennzahlen orientiert, ${ }^{20}$ sondern auf eine weitere Öffnung der Märkte und eine konsequentere Umsetzung des Binnenmarktprogramms abzielt. Insofern mag die Hoffnung begründet sein, dass die Wirtschafts- und Finanzkrise Auslöser und Verstärker für weitere Bemühungen in diesem Bereich sein könnte. Hierzu zählen insbesondere die konsequente Schaffung binnenmarktähnlicher Verhältnisse in den Bereichen Infrastruktur und Netzindustrien, die Erhöhung der Mobilität des Produktionsfaktors Arbeit sowie die fortschreitende Umsetzung des Binnenmarktes im Dienstleistungssektor. ${ }^{21}$ Hier sollten für die Zukunft stärkere Fortschritte erzielt werden. Die Forderung nach der Realisierung des Binnenmarktes in allen Bereichen ist hier mit Nachdruck zu unterstützen.

Daher zielen die Lehren, die aus der Krise für die Wirtschaftspolitik im realen Sektor der Europäischen Union gezogen werden können, primär auf die Stärkung der wirtschaftlichen Wachstumskräfte ab. Seriöserweise vermag zum gegenwärtigen Zeitpunkt noch niemand genau zu prognostizieren, wie lang die rezessive Entwicklung noch anhält beziehungsweise wann sie endgültig überwunden ist. Daher kann die langfristige Strategie auf Gemeinschaftsebene auch nur eine auf die Stärkung des Binnenwachstums ausgerichtete Wirtschaftspolitik sein. Neben der schon angesprochenen Stärkung des Binnenmarktes könnte auch eine reformierte und richtig praktizierte Lissabon-Strategie Erfolg versprechend sein. Dies setzt aber voraus, dass man sich von der in einigen Mitgliedstaaten immer noch vorherrschenden Dominanz einer strukturkonservierenden sektoralen Wirtschaftspolitik, wie sie beispielsweise durch die Vergabe von Beihilfen im Automobilsektor zum Ausdruck kommt, verabschiedet und stärker die Möglichkeiten einer ergebnisoffenen prozessorientierten Innovationspolitik nutzt. Vor allem wäre eine weitere Förderung in den Bereichen Bildung und Grundlagenforschung erforderlich.

Eine wesentliche Hoffnung ist, dass die europäischen Institutionen und die Mitgliedstaaten die sich jetzt bietenden Möglichkeiten zu einer echten Reform der Lissabon-Strategie nutzen. Die daraus resultierenden Wachstumskräfte könnten der Europäischen Union nicht

19 Rat der Europäischen Union: Schlussfolgerungen des Vorsitzes. Europäischer Rat (Lissabon) 23. und 24. März 2000, SN 100/00.

20 Europäische Kommission: Moving Europe's productivity frontier. The EU economy: 2007 review, European Economy 8/2007.

21 Siehe hierzu bereits André Sapir u.a.: An Agenda for a Growing Europe. Making the EU Economic System Deliver, Brüssel 2003. 
nur helfen, die gegenwärtige Wirtschaftskrise zu überwinden, sondern dauerhaft ihre eigene Wettbewerbsfähigkeit zu steigern.

Fragt man nach den entsprechenden zukünftigen Risiken, die sich für die Wirtschaftspolitik der Mitgliedstaaten ergeben, so ist hier insbesondere auf die erhebliche Verschlechterung der Staatsfinanzen für die nächsten Jahre zu verweisen. Dabei wird es von entscheidender Bedeutung sein, dass die Mitgliedstaaten spätestens mit Beginn der konjunkturellen Erholung ihre strukturellen Defizite verringern. Das heißt: Der finanzpolitische Handlungsspielraum wird für alle Mitgliedstaaten stark eingeschränkt sein. Dies gilt umso mehr, als auch in der nahen Zukunft keine allzu hohen realen Wachstumsraten des Bruttoinlandsprodukts erwartet werden können. ${ }^{22}$ Gerade vor diesem Hintergrund gewinnt die Implementierung einer nachhaltigen Wachstumsstrategie an Bedeutung. Aufgabe der europäischen Institutionen wird es dabei sein, einerseits die Anreize zu einer tragfähigen Konsolidierung der nationalen Staatsfinanzen zu erhöhen und die Glaubwürdigkeit des Stabilitäts- und Wachstumspaktes zu stärken sowie andererseits mittels einer wachstumsorientierten Binnenmarktpolitik die Konsolidierungsanstrengungen der Mitgliedstaaten zu unterstützen. Die Zukunft der Europäischen Union wird insbesondere davon abhängen, inwieweit es den Institutionen gelingt, diese Aufgabe zu lösen.

22 Europäische Zentralbank: Entwicklung der öffentlichen Finanzen, in: Europäische Zentralbank (Hrsg.): Monatsbericht September 2009, Frankfurt am Main 2009, S. 88-97, hier S. 92. 